\title{
ONTOLOGY-BASED SEMANTIC CLASSIFICATION OF SATELLITE IMAGES: CASE OF MAJOR DISASTERS
}

\author{
Hafidha Bouyerbou ${ }^{13}$, Kamal Bechkoum ${ }^{2}$, Nadjia Benblidial, Richard Lepage ${ }^{3}$ \\ ${ }^{1}$ School of Sciences, University of Blida, \\ Blida, Algeria \\ h. bouyerbou@univ-blida.dz \\ ${ }^{2}$ School of Science and Technology, University of Northampton \\ Northampton, United Kingdom \\ ${ }^{3}$ Ecole de Technologie Supérieure \\ Montreal, Quebec, Canada
}

\begin{abstract}
The International Charter" "Space and Major Disasters" is regularly activated during a catastrophic event and offers rescue team damage maps. Most of these maps are built by means of satellite image manual processing, which is often complex and demanding in terms of time and energy. Automatic processing offers prompt treatment; nevertheless it usually presents a semantic gap handicap. The exploitation of ontologies to bridge the semantic gap has been widely recommended due to their quality of knowledge representation, expression, and discovery. In this work, we present an ontology-based semantic hierarchical classification method to undertake this problem. Ontology components are translated to image-based parameters and used to assist the classification process with two levels and 12 embedded classes. The region of interest is selected from the first level, and exhaustively analyzed and classified at the second level. The 2010 Haiti earthquake was selected as study area for this work. Experiments were performed using very high resolution multi-temporal QuickBird imagery and eCognition software.
\end{abstract}

Index Terms - Major disaster, semantics, ontology, satellite image, classification.

\section{INTRODUCTION}

Increasing number of natural and man-made disasters such as earthquake, tsunami, forest fire, and flood, prompted various organizations to make more effort in the disaster management field in order to save more lives. Finding and integrating consistent data and information related to a disaster in a quick manner is a critical step for effective decision making. The integration of remote sensing data can provide important knowledge, facilitate the relief task, and guide teams on the ground in their damage assessment.

The design of successful image analysis systems requires knowledge about the underlying problem solving processes. The better the knowledge about the process and the better this knowledge can be represented in the system, the more useful the extracted information will be [1].
Ontologies have been selected as one of the most powerful knowledge representation techniques. In the recent years, ontologies become one of the most important areas of interest in the geographic information science. The use of ontologies to bridge the semantic gap has been widely recommended due to their quality of knowledge representation, expression, and discovery. Directly or indirectly, ontologies specify the composition, structure, and basic properties of the simplified worlds that our models represent, and clarify the intended meanings of the terms we use [2].

This work brings a solution to identify hidden knowledge and bridge the gap between the results of automatic classification techniques, and high level semantics using human-defined ontologies.

We will describe in the following an ontology-based semantic hierarchical classification method and its application on multi-temporal QuickBird imagery. The remainder of this paper is structured as follows. We present in section 2 the study area and material. Our methodology is described in section 3. Results and discussion are provided in section 4, and finally, conclusion is given in section 5 .

\section{STUDY AREA AND MATERIAL}

\subsection{Study Area}

The 2010 Haiti earthquake was selected as event study area for this work, precisely, the Port-au-Prince region. Haiti was selected as a study area since multiple pre and post-disaster images were made available by various providers. We exploit in this work pre-disaster pansharpened Quickbird images acquired in February 2009, almost a year before the earthquake, and post-disaster Quickbird images of the same area acquired in January 2010, a few days after the earthquake. Fig. 1 shows a multi-temporal region of the subset, where (a) illustrates the pre-disaster, and (b) the post-disaster region.

\footnotetext{
${ }^{1} \mathrm{http}: / /$ www.disasterscharter.org/ 


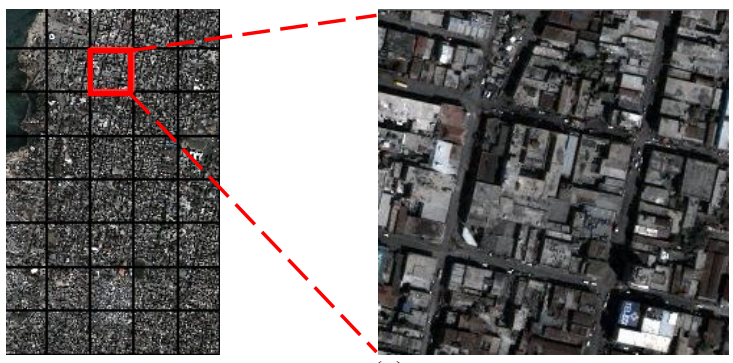

(a)

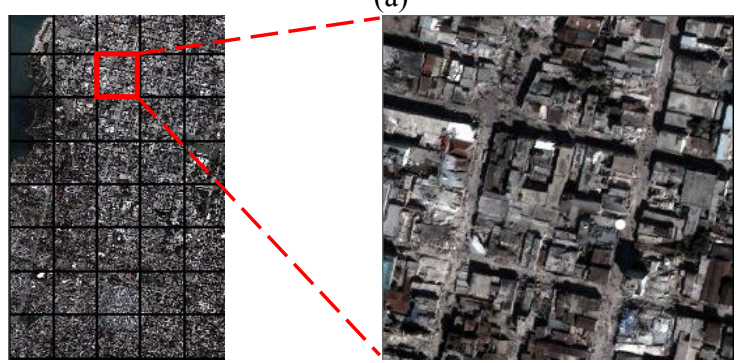

(b)

Fig. 1. Multi-temporal Port-Au-Prince (Haiti) subset regions

\subsection{Data and Software}

A four-band (R, G, B, NIR) pansharpened multispectral QuickBird standard image (resolution $0.6 \mathrm{~m}$, acquisition date February 22, 2009), and a post-disaster four-band (R, G, B, NIR) pansharpened multispectral QuickBird standard image of the same area (resolution $0.6 \mathrm{~m}$, acquisition date January 15, 2010), were combined in this work.

Protégé 4.3 software was used for ontology development, and the eCognition Developer 8.7 software system was used to conduct image segmentation, classification, and change detection.

\section{METHODOLOGY}

Our methodology is described in Fig. 4. Initially, a geographic ontology was developed to assist the classification process. The ontology components are translated to imagebased parameters to use in classification. Only two levels of the ontology with 12 embedded classes (see Fig. 2) were needed in this work. Shadow class was added for class discrimination.

First of all, lower resolution images are created from the original very high resolution multi-temporal images and classified, after performing a multi-resolution segmentation, into three classes using ontology first level. The class of interest at level 1 is the one that can be affected by the disaster. As the disaster in this case is earthquake; the damaged structures are mainly buildings and roads. Thus, Artificial Surface is selected as region of interest. As next step, the region of interest resolution is increased to the original resolution. Multi-resolution segmentation is performed at each level for a specific class with new scale and parameters. Independent classification of the multi-temporal images is performed using ontology second level. Finally the two classifications results are combined and an object-based comparison is performed for change detection.

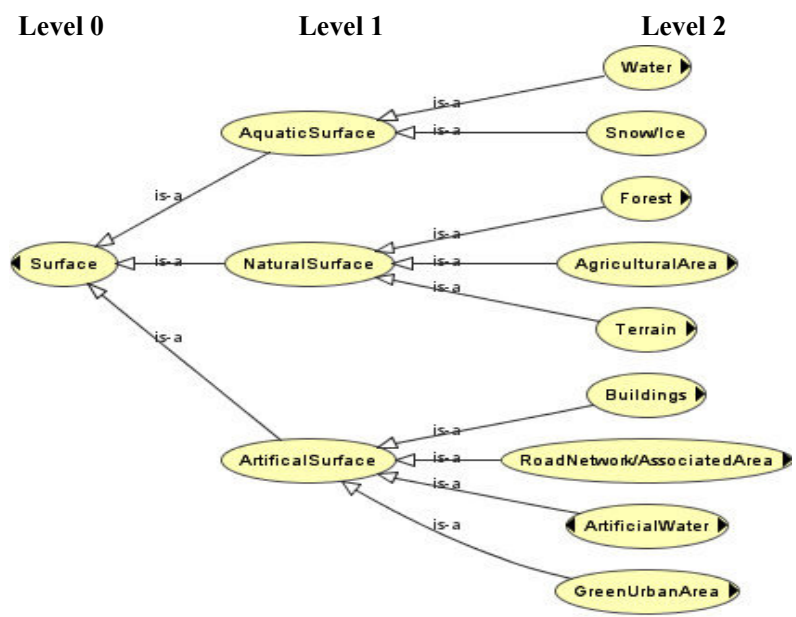

Fig. 2. Ontology levels and classes used in the classification

\subsection{Domain-Ontology development}

An OWL geographic ontology for major disasters named GEO-MD (Geographic Ontology for Major Disasters) was developed using Protégé 4.3 and OWL-DL (Ontology Web Language Description Logics), to handle semantics problems in geographic data, provide a referential geographic taxonomy in the context of major disasters, and assist the semantic classification of satellite images at various scales.

GEO-MD consists of three parts: surface, disaster and damage. These are jointly gathered with semantic, temporal, and spatial relationships. Surface mainly includes geographical concepts, with five hierarchical levels. Disaster includes concepts of major disasters divided into two main classes: Manmade and Natural disasters. Only disasters included in the International Charter "Space and Major Disasters" have been taken into account in our work. Damage contains concepts of the damage following a disaster. Two damage classes were defined: Land cover damage, which covers damage to the ground cover, and Material damage, which includes structural damage.

Interaction between the three sub-ontologies will guide the semantic classification by defining the damage category, reducing the area of interest in the satellite image, etc. We translated ontology concepts and relationships into imagebased parameters (see Fig. 3), and defined the corresponding rule sets for the classification progress and refinement.

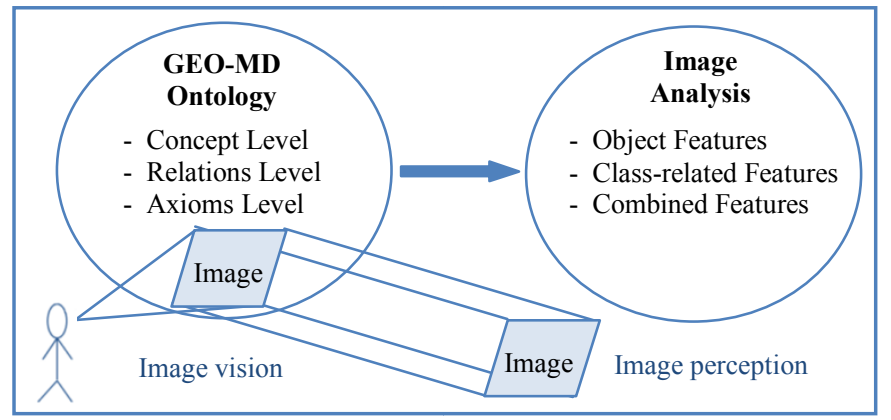

Fig. 3. Translation of the ontology into image-based parameters 


\subsection{Multiresolution Segmentation}

The segmentation algorithm used in eCognition is a regiongrowing method where pixels whose attribute values represent a region are collected. The multiresolution algorithm adopted in this step is a bottom up region-merging technique proposed by Baatz and Schäpe [3] in which, for a d-dimensional feature space $f$, the "degree of fitting" $\mathrm{h}$ is defined as:

$$
h=\sqrt{\sum_{d}\left(f_{1 \mathrm{~d}}-f_{2 \mathrm{~d}}\right)^{2}}
$$

This distance can be furthermore standardized by the standard deviation over the feature segments in each dimension:

$$
h=\sqrt{\sum_{d}\left(\frac{f_{1 \mathrm{~d}}-f_{2 \mathrm{~d}}}{\sigma_{f d}}\right)^{2}}
$$

Fig. 5 (a) shows segmentation results at level 1 with scale 100 , shape 0.2 , and compactness 0.5 . Image resolution was reduced to $8 \mathrm{~m}$. Segmentation results at level 2, with image resolution $0.6 \mathrm{~m}$, are shown in Fig. 5 (b) with scale 32, shape 0.1 , and compactness 0.5 .

\subsection{Multi-level classification}

At level 1, Normalized Difference Vegetation Index (NDVI) was employed to extract Natural Surface, Normalized
Difference Water Index (NDWI) to extract Aquatic Surface, and Built-up Area Index (BAI) to extract Artificial Surface. NIR band and contextual features are utilized for correction and refinement. Level 1 classification results are used to select the region of interest for the classification at level 2 for both pre and post-disaster images.

$$
\begin{aligned}
N D V I & =\frac{\mathrm{NIR}-\mathrm{RED}}{\mathrm{NIR}+\mathrm{RED}} \\
N D V I & =\frac{\mathrm{G}-\mathrm{NIR}}{\mathrm{G}+\mathrm{NIR}} \\
B A I & =\frac{\mathrm{B}-\mathrm{RED}}{\mathrm{B}+\mathrm{RED}}
\end{aligned}
$$

At Level 2, Green Urban Area is first extracted based on NDVI index in equation (3) with a threshold set to 0.2. Some shadow was misclassified at this level; a threshold for NIR is set inferior to 30 to distinguish shadow from Green Urban Area. The remaining shadow is farther classified with low brightness and density thresholds. No Artificial Water was detected in our dataset.

After creating vegetation and shadow mask, only Buildings, and Roads and Associated Area remain to be classified. However, these two classes are very similar in terms of spectral information; it is difficult to classify them properly by spectral features only [4].

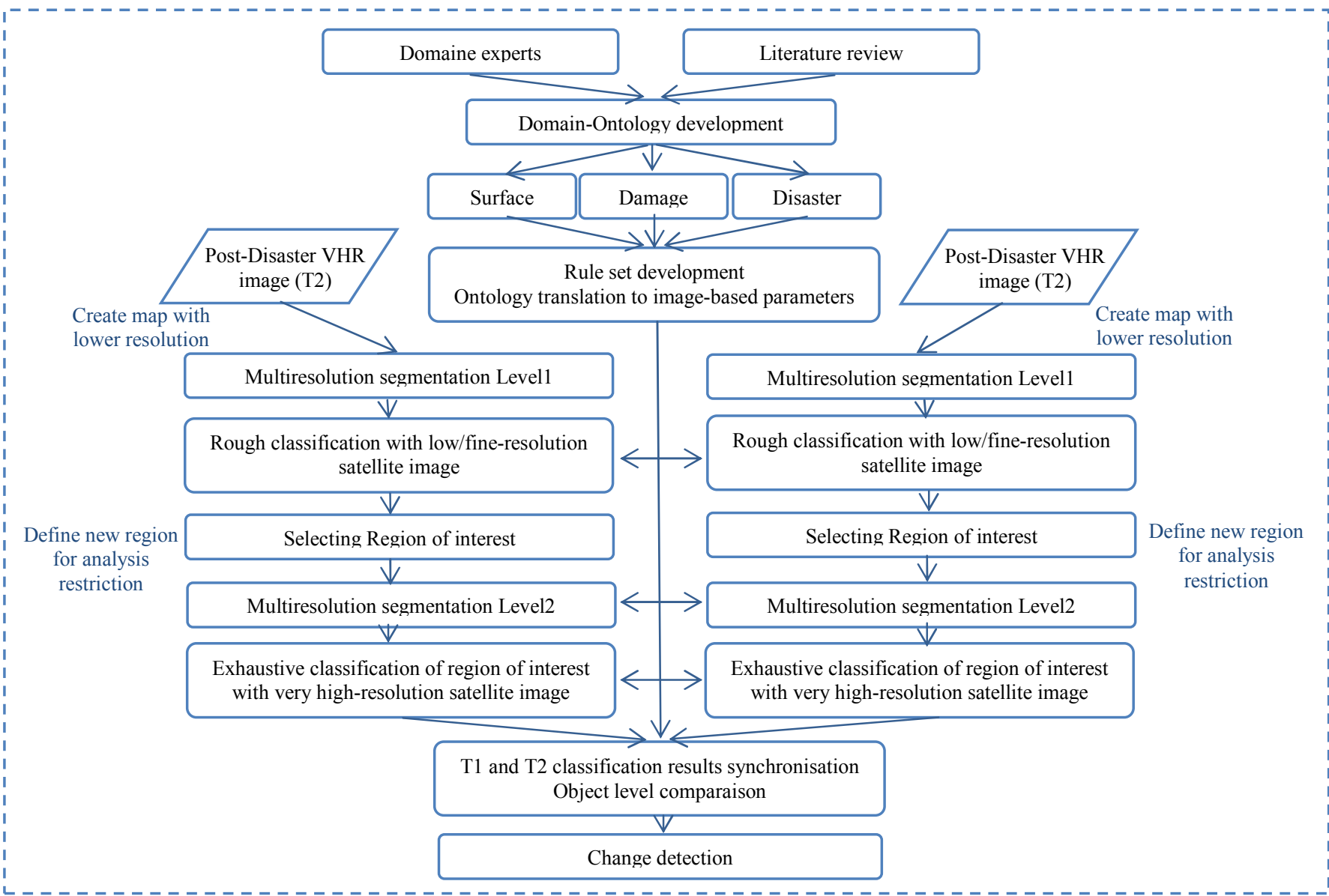

Fig. 4. Methodology overview 


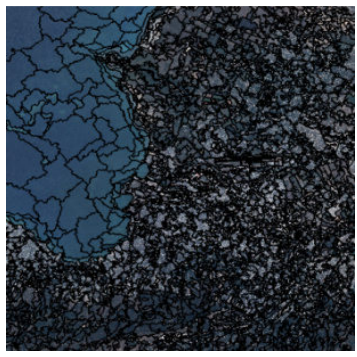

(a)

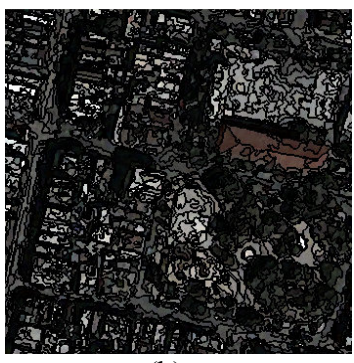

(b)
Fig. 5. Multiresolution segmentation

Gray Level Co-occurrence Matrix (GLCM) and Gray Level Difference Vector (GLDV), in addition to a set of geometric features (e.g. pixel area, length/width, rectangular fit, and elliptic fit), and class-related features (e.g. border to, overlap of two object, and distance), which were formerly translated from the ontology, are used at this level to separate the two classes.

\subsection{Change detection}

Two classes were the focus of this step: Buildings, and Roads and associated area. A mask of Green Urban Area (Vegetation) and Shadow is created for T1 (pre-disaster dataset) and T2 (post-disaster dataset) maps. After classification, T1 map Buildings, and Roads and Associated Area classification results are synchronized on T2 map. Object-level comparison is performed for each object of these two classes and changes are highlighted.

\section{RESULTS AND DISCUSSION}

Multi-temporal pansharpened QuickBird imagery was chosen for experimental results. Fig. 6 (a) and (c) show the original QuickBird images with different resolution $(8 \mathrm{~m}$ and $0.6 \mathrm{~m}$ respectively). Fig. 6 (b) shows the classification results at Level 1, and Fig. 6 (d) shows the classification results at Level 2. Ontology-driven geometric features and classrelated features have clearly improved the classification results using only multispectral information. The nature of buildings and roads in Haiti was a drawback in this work as they are not well structured which did not allow us to go deeper in the ontology levels.

\section{CONCLUSION}

This paper brings a solution to identify hidden knowledge and bridge the gap between the results of stateof-the-art automatic classification techniques, and high level human semantics using human-defined ontologies. The high-level semantic represented by the ontology noticeably improves the automatic classification results, and lead to automatically understand and describe remote sensing data.

Work is still in progress, and more experiments on postdisaster imagery and change detection are compulsory. Nevertheless, we were able to present preliminary classification results that show the high potential of the ontology-based method.

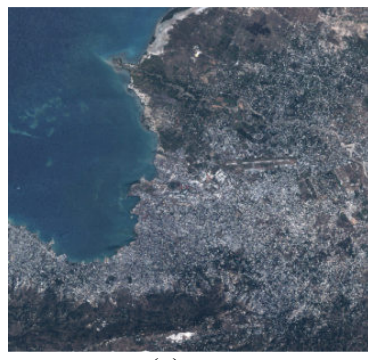

(a)

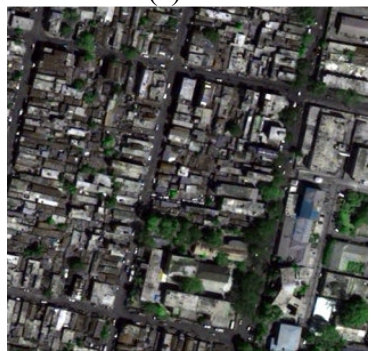

(c)

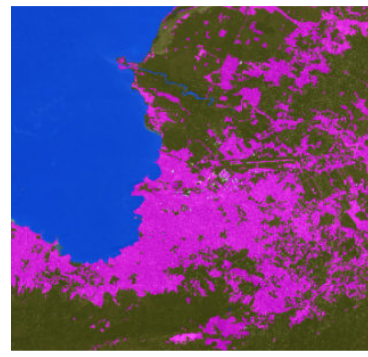

(b)

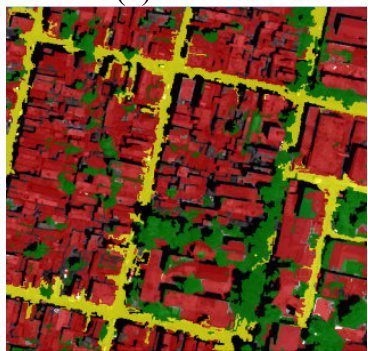

(d)

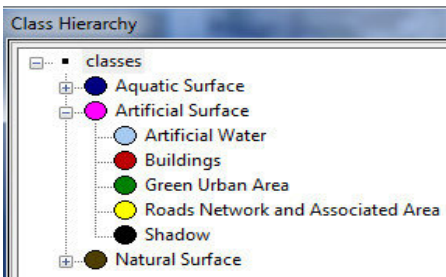

Fig. 6. Multi-level classification results

The use of LIDAR data, and metadata (e.g. road network maps, city map), can improve the classification accuracy and allow us to explore more ontology levels by giving additional information. GEO-MD application on other disaster classes (i.e. flood, and forest fire), change detection, and damage assessment, are subject to our future work.

\section{ACKNOWLEDGMENT}

This research was partially funded by Effigis GeoSolutions. We wish to thank CSR, University of Texas, and Digital Globe for providing pre and post-disaster imagery.

\section{REFERENCES}

U. C. Benz, P. Hofmann, G. Willhauck, I. Lingenfelder et M. Heynen, "Multi-resolution, object-oriented fuzzy analysis of remote sensing data for GIS-ready information," ISPRS Journal of photogrammetry and remote sensing, vol. 58, pp. 239-258, 2004.

[2] H. Couclelis, "Ontologies of geographic information," International Journal of Geographical Information Science, vol. 24, pp. 1785-1809, 2010/11/26 2010.

[3] M. S. Baatz, A., , "Multiresolution Segmentation - an optimization approach for high quality multi-scale image segmentation," In: Strobl, J. et al. (eds.): Angewandte Geographische Informationsverarbeitung XII. Wichmann, Heidelberg, 2000.

[4] Y. Chen, W. Su, J. Li et Z. Sun, "Hierarchical object oriented classification using very high resolution imagery and LIDAR data over urban areas," Advances in Space Research, vol. 43, pp. 1101-1110, 4/1/ 2009. 